Results A diagnosis of COPD was significantly associated with a two- to three-fold increased risk of CV mortality compared to people without COPD. Additionally, in patients with COPD there was a significantly increased incident risk of up to almost four-fold for hypertension (rate ratio [RR]: 0.95; 95\% confidence interval $0.91-0.99$ to $3.57 ; 3.41-3.74)$, up to sixfold for heart failure (RR: $1.46 ; 1.38-1.53$ to 5.94 ; $5.50-$ 6.42), up to five-fold for dysrhythmia (RR: $1.19 ; 0.98-1.43$ to $4.74 ; 4.27-5.26$ ), and up to two-fold for AMI (RR: 1.18; $0.81-1.71$ to $1.89 ; 1.71-2.09)$. An increased risk was also observed for stroke (hazard ratio: $1.30 ; 1.18-1.43$ ) and diabetes (risk ratio: 1.25; 1.16-1.34). No data on incident risk were identified for hypercholesterolaemia. A significantly increased risk in $\mathrm{CV}$ mortality compared to patients without COPD was demonstrated across all severity categories of mild, moderate and severe COPD; the risk increased further with increasing severity of COPD.

Conclusions There is substantive evidence for an association between COPD and the incidence of specific cardio- and cerebrovascular acute events or diseases. To minimise the risk of poor outcomes, it is important to ensure that patients with comorbid COPD and CVD are diagnosed early and optimally managed at all levels of health-care settings.

Please refer to page A190 for declarations of interest related to this abstract.

\section{S101 METHODS FOR ASSESSING THE SUCCESS OR FAILURE OF COPD EXACERBATION TREATMENTS IN THERAPEUTIC CLINICAL TRIALS: A META- EPIDEMIOLOGICAL SYSTEMATIC REVIEW}

\footnotetext{
${ }^{1,2} \mathrm{AG}$ Mathioudakis, ${ }^{3} \mathrm{~S}$ Ananth, ${ }_{4}^{4} \mathrm{~T}$ Bradbury, ${ }^{5} \mathrm{~B}$ Csoma, ${ }^{6} \mathrm{G}$ Fernandez Romero, ${ }^{6} \mathrm{G}$ Criner, ${ }^{4} \mathrm{C}$ Jenkins, ${ }^{7} \mathrm{PR}$ Williamson, ${ }^{8,9} \mathrm{~J}-\mathrm{U}$ Jensen, ${ }^{1,2} \mathrm{~J}$ Vestbo, for the DECODE-NET. ${ }^{1}$ The University of Manchester, Manchester Academic Health Science Centre, Manchester, UK; ${ }^{2}$ Manchester University NHS Foundation Trust, Manchester, UK; ${ }^{3}$ West Hertfordshire Hospitals NHS Trust, Watford, UK; ${ }^{4}$ University of New South Wales, Sydney, Australia; ${ }^{5}$ Semmelweis University, Budapest, Hungary; ${ }^{6}$ Lewis Katz School of Medicine, Philadelphia, Pennsylvania, USA; ${ }^{7}$ University of Liverpool (a member of Liverpool Health Partners), Liverpool, UK; ${ }^{8}$ HerlevGentofte Hospital, Hellerup, Denmark; ${ }^{9}$ University of Copenhagen, Copenhagen, Denmark
}

\subsection{6/thorax-2021-BTSabstracts. 107}

Introduction The overall outcome of a COPD exacerbation (treatment success or failure) is a critical outcome for clinical trials evaluating the management of COPD exacerbations. However, trials use heterogeneous definitions and instruments to evaluate this outcome, limiting the comparability of trial results. Here, we describe how often different measurement instruments are used to evaluate the overall outcome of COPD exacerbations, aiming to promote consistency.

Methods MEDLINE and the Cochrane Airways Trial Register were systemically searched. COPD exacerbation trials (20062020) reporting on treatment success or failure were included. Risk of bias was assessed. The definitions and measurement instruments used to assess treatment success/failure were collected and described narratively.

Results 56/176 (31.8\%) of COPD exacerbation trials assessed the overall outcome of the exacerbation (treatment success or failure). 9/56 (16.1\%) of studies had a low risk of methodological bias. Two categories of outcomes evaluating treatment success or failure were identified. Twenty-four RCTs used composite endpoints consisting of several undesirable outcomes to define treatment failure. The most frequently used components were death (59\%), hospital admission (52\%),
Abstract S101 Table 1 Frequently used definitions of various COPD exacerbations states

\begin{tabular}{ll}
\hline Cure or Resolution & $\begin{array}{l}\text { Number of Studies } \\
\text { ( } \mathbf{n}=\mathbf{5 6})\end{array}$ \\
\hline $\begin{array}{l}\text { Complete resolution of all signs and symptoms of the exacerbation. } \\
\text { Sufficient improvement of the signs and symptoms such that no } \\
\text { additional systemic treatments were prescribed. }\end{array}$ & 5 \\
$\begin{array}{l}\text { Improvement } \\
\text { Improved signs and symptoms, without any new signs or }\end{array}$ & 8 \\
symptoms. & 6 \\
Improved symptoms as evaluated by clinical scores. & \\
Treatment failure & 7 \\
Lack of resolution of signs and symptoms, requiring additional \\
treatment, or death. \\
$\begin{array}{l}\text { Persistence or worsening of signs and symptoms, or death. } \\
\text { Lack of resolution of signs and symptoms, or need for further } \\
\text { treatment. }\end{array}$ \\
\hline
\end{tabular}

treatment intensification (52\%) or mechanical ventilation (37\%). By comparison, thirty-three RCTs used qualitative descriptions of the status of the exacerbation (such as cure, improvement or failure), which were based on the patients' symptoms and signs (table 1). The overall outcome was evaluated at different timepoints, between 2 hours and 1 year from presentation.

Conclusions There is significant heterogeneity in the instruments used to evaluate treatment success or failure in COPD exacerbation trials. Standardization could promote comparability.

\section{S102 THE UNDER RECOGNISED ROLE OF MODERATE EOSINOPHILIA ON EXACERBATION FREQUENCY IN COPD PATIENTS: A SINGLE CENTRE STUDY}

P Dobson, A Dewar. Guy's and St Thomas' Hospitals, London, UK

\subsection{6/thorax-2021-BTSabstracts. 108}

Introduction and Objectives High eosinophil levels in patients with COPD are a well established risk factor for frequent exacerbations. Our study aimed to determine whether more moderate eosinophil levels are also a significant driver of frequent exacerbations.

Methods We retrospectively identified 213 patients who presented with a severe exacerbation of COPD to our unit from 01 January 2018 to 31 December 2018. Patients were divided into three groups: high eosinophilia (peak eosinophil level $\geq 0.5$ ); moderate eosinophilia (peak eosinophil level 0.3-0.4) and low eosinophilia (peak eosinophil level $\leq 0.2$ ).

The exacerbation frequency and trajectory of patients was followed up for 18 months. Standard univariate statistical analysis was undertaken to determine the impact high, moderate, and low eosinophilia had on exacerbation frequency.

Results For patients who were initially infrequent exacerbators, moderate eosinophilia was associated with a shift towards increased exacerbation frequency $(p<0.05)$. High eosinophilia however was not associated with increased exacerbation frequency $(p=0.897)$. 